\title{
Identifying threading dislocations in CdTe films by reciprocal space mapping and defect decoration etching
}

\author{
Mustafa Polat, ${ }^{\text {a) }}$ Elif Bilgilisoy, ${ }^{\text {b) }}$ Ozan Arı, Orhan Öztürk, and Yusuf Selamet ${ }^{\mathrm{c})}$ \\ Department of Physics, Izmir Institute of Technology, Izmir 35430, Turkey
}

(Received 13 February 2018; accepted 10 August 2018; published online 31 August 2018)

\begin{abstract}
We study threading dislocation (TD) density of high-quality cadmium telluride (CdTe) layers grown on a (211) oriented GaAs substrate by molecular beam epitaxy. High-resolution X-ray diffraction was performed to calculate the density of screw-type TDs by measuring the broadening of the asymmetrical (511) Bragg reflections of CdTe epilayers. In addition, total TD densities were determined by the Everson-etching method and were compared with screw TDs. Our results show that the total TD densities in CdTe films were dominated by those with screw character. The screw component TDs are estimated to account for more than $90 \%$ of the total TD density. CdTe layers grown at a thickness of less than $3.0 \mu \mathrm{m}$ typically exhibit the screw TD densities in the $10^{6} \mathrm{~cm}^{-2}$ and $10^{7} \mathrm{~cm}^{-2}$ range. It can be noted that as the nucleation temperature increases, i.e., $\geq 222^{\circ} \mathrm{C}$, both the area density of TDs with the screw component of the CdTe films and the total TD density are roughly four times larger than those of the epilayer grown at the nucleation temperature of $215^{\circ} \mathrm{C}$. Furthermore, we discuss the influence of the II/VI flux ratio on the density of threading dislocations. The contribution of screw TDs to the total TD density showed a significant decrease in roughly $30 \%$ in the case of a high II/VI flux ratio. We further examine the reciprocal space maps in the vicinity of the (422) reflections. Published by AIP Publishing.

https://doi.org/10.1063/1.5025782
\end{abstract}

\section{INTRODUCTION}

Cadmium telluride (CdTe) grown on a highly latticemismatched GaAs substrate has attracted significant attention in various fields, ranging from low cost mercury cadmium telluride (MCT) infrared detectors, ${ }^{1,2}$ solar cells ${ }^{3}$ to nuclear radiation detectors. ${ }^{4}$ Although remarkable progress has been achieved in epitaxial growth of CdTe thin films in recent years, ${ }^{5}$ the lattice constant mismatch of approximately $14 \%$ at room temperature and the large difference in thermal expansion coefficients ${ }^{6}$ still present considerable challenges for researchers. It is well known that latticemismatched epitaxial growth leads to the formation of a high density of misfit dislocations (MDs) at the interface and a large number of threading dislocations (TDs) propagating into CdTe epilayers, thereby significantly degrading the quality of the heterostructure. ${ }^{7}$ Both screw and edge components of TDs exist in this epilayer as evidenced by various techniques. $^{7-9}$ However, in comparison with the edge TDs, the screw TDs are much more likely to have a higher density due to their lower dislocation energies according to a recent theoretical study. ${ }^{10}$ This inevitably drives a need to study and to determine the density of screw TDs in the epilayers in much more detail and greater precision.

Mainly, defect decoration etching and cross-sectional transmission electron microscopy (TEM) are used to reveal and to observe TDs. However, these two methods can permanently damage the analyzed films. Therefore, a non-

\footnotetext{
${ }^{a)}$ Electronic mail: mustafapolat@iyte.edu.tr

${ }^{b)}$ Present address: Department of Chemistry and Pharmacy, FriedrichAlexander-Universität Erlangen, Nürnberg 91054, Germany.

${ }^{c)}$ Deceased 5 August 2016.
}

destructive tool would be beneficial to measure the high defect densities in CdTe epitaxial layers. One such tool is high-resolution X-ray reciprocal space mapping (RSM) to study structural defects of such heterostructures. ${ }^{11,12}$ Actually, there is a reciprocal-space model to analyze the highly distorted epitaxial thin films. ${ }^{13}$ The physics behind this model is to estimate defect-induced broadening of reciprocal lattice points (RLPs). ${ }^{13,14}$ The reason for the broadening is that the strain field around the dislocations gives rise to a partial loss of the long-range order of the crystal lattice, as well as to the nonuniform dilatation and the rotation of the crystal planes. ${ }^{15}$ In the model, ${ }^{13,14}$ the epilayer is composed of single crystallites that are known as mosaic blocks, each with certain mean vertical and lateral dimensions. The average extension of these small single crystalline regions is defined as a coherence length in which X-rays are scattered coherently, with the assumption that these crystalline regions are free of defects. Coherence length perpendicular (parallel) to the plane of growth corresponds to the vertical (lateral) coherence length. Similarly, the out-of-plane rotation of the crystal planes perpendicular to the surface normal refers to the mosaic tilt, while the in-plane rotation around the surface normal can be characterized by means of the mosaic twist. ${ }^{13-15}$ The broadening of the RLPs must be examined to determine the four characteristic parameters. The presence of the edge components of TDs leads to the twist, and skew sample geometry can be utilized to find out both the twist and the vertical coherence length. ${ }^{13,14}$ Since screw TDs are the dominant type of dislocations in this epilayer as mentioned previously, we only employed traditional coplanar scans in this study to evaluate the tilt and the lateral coherence length. ${ }^{16,17} \mathrm{Next}$, the density of TDs with 
screw-component character was estimated with a formula that employs a tilt parameter. ${ }^{18-20}$

Although a quantitative estimation of the density of TDs in epitaxial III-V layers has been of significant interest in the last few decades due to the nondestructive nature of the RSM technique, ${ }^{11}$ II-VI materials have suffered from a lack of high-quality studies associated with this issue. ${ }^{21}$ A limited number of studies have been conducted so far to investigate the structural properties of $\mathrm{CdTe} / \mathrm{GaAs}$ heterostructures by X-ray diffraction (XRD) techniques. The dependence of the structural properties on the growth temperatures, ${ }^{22}$ the effect of the residual stresses, ${ }^{6}$ the role of thermal expansion matching, ${ }^{23}$ the lattice tilting of CdTe epilayers, ${ }^{24}$ and fullwidth at half-maximum (FWHM) values of the X-ray double-crystal rocking curve (XDRC) measurements ${ }^{25}$ were studied in real space. In our previous study, ${ }^{26}$ the screw TDs and shear strain coexisting in the CdTe epilayer were evaluated with the help of RSM. However, we believe that the RSM technique, when combined with other techniques such as the defect decoration etching, may be a more promising approach for predicting not only the density of screw TDs but also percentage of TDs with screw components in CdTe films.

In this study, motivated by the approach suggested by Srikant et al., ${ }^{13}$ the densities of screw-component TDs in a series of CdTe epilayers were estimated by measuring the broadening of the Bragg peaks using high resolution X-ray reciprocal space mapping. These CdTe layers were grown on epi-ready GaAs(211)B (extremely low contamination and As terminated) substrates using molecular beam epitaxy (MBE) under different growth conditions. In addition, we performed the Everson-etching method to determine the densities of the total etch pit density (EPD). Atomic force microscopy (AFM) and scanning electron microscopy (SEM) were conducted to examine the surface structure of the CdTe thin films before and after defect decoration etching, respectively. The densities of screw TDs, which were extracted from the amount of broadening of the reciprocal lattice points, were compared with the densities of the total etch pit density. In this context, a detailed discussion of the growth condition effect on the dislocation density in (211)-oriented CdTe samples was reported. Furthermore, spatial distributions of the screw TD densities across the entire samples under study were obtained with the help of an XY mapping technique. These XY maps, presented in the form of polar contour plots, not only provide useful information for the distribution of the densities of screw TDs but are also important to demonstrate the shapes and the sizes of CdTe epilayers. Moreover, the measured RSMs in the vicinity of the symmetric (422) reflections yield evidence for the presence of mosaicity in the films.

\section{EXPERIMENTAL PROCEDURE}

\section{A. MBE growth of CdTe epilayers on (211) GaAs substrates}

A series of CdTe epilayers were grown on 3-in. semiinsulating (211)B GaAs substrates (B refers to the As terminated surface) using a Veeco GEN20MZ MBE system operated in a class 1000 cleanroom environment. The (211) surface composed of the (111) terraces and the (100) edges is suitable to achieve a step-flow growth mechanism that leads to reduce twinning and antiphase domain formation for as-grown specimens. ${ }^{27}$ Prior to the CdTe thin film growth, the protective oxide layers on $\mathrm{GaAs}$ substrates were thermally desorbed between $604{ }^{\circ} \mathrm{C}$ and $613^{\circ} \mathrm{C}$ deoxidation temperatures. To maintain surface polarity, the deoxidation steps were carried out under an $\mathrm{As}_{4}$ flux. After the desorption step, thin nucleation layers of CdTe $(\sim 90 \mathrm{~nm})$ were deposited at about $220^{\circ} \mathrm{C}$ in order to suppress the formation of twinning defects and to preserve the (211) orientation. The growth of thick CdTe epilayers has been performed at about $300{ }^{\circ} \mathrm{C}$ under a $\mathrm{Te}_{2}$ flux and at a rate of about $1 \mu \mathrm{m} / \mathrm{h}$. The thickness of the CdTe films varied from $2.7 \mu \mathrm{m}$ to $3.0 \mu \mathrm{m}$. A precise non-contact temperature measurement of (211)B CdTe epilayers was conducted using a calibrated pyrometer system. We acquired flux ratios by measuring beam equivalent pressures (BEPs) of $\mathrm{Te}_{2}$ and CdTe fluxes during the growth with respect to the base pressure of the MBE growth chamber. Table I summarizes the growth parameters of selected specimens. In this table, the II/VI flux ratio is the relationship between the total amount of Te and Cd elements.

As an in-situ characterization technique, reflection highenergy electron diffraction (RHEED) was used to monitor the structural evolution and the surface crystallinity during the growth. A Woollam model M-2000× spectroscopic ellipsometer (SE) was used as an ex-situ tool to measure epilayer thicknesses. In order to determine the thicknesses, the ellipsometric data were fitted using a three-layer optical model (oxide/CdTe/GaAs). Thicknesses of CdTe epilayers were also obtained from the intensity oscillations in the transmittance spectra using Fourier transform infrared spectroscopy (FTIR). The transmittance of analyzed films was measured at room temperature in the range from $4000 \mathrm{~cm}^{-1}$ to $15500 \mathrm{~cm}^{-1}$ using a Bruker Vertex-80v spectrometer equipped with $\mathrm{KBr}$ windows.

\section{B. Everson-etching method}

After the growth, the specimens with a diameter of 3 in. were diced into two half-discs. One half was analyzed using the RSM technique. The other half was again cut into several small pieces and then the standard Everson EPD method was applied to some of these pieces. The Everson etch solutions were prepared with volume ratios of lactic acid $\left(\mathrm{C}_{2} \mathrm{H}_{4} \mathrm{OHCOOH}\right)$ :nitric acid $\left(\mathrm{HNO}_{3}\right)$ :hydrofluoric acid $(\mathrm{HF})$ of $25: 4: 1$ in polypropylene

TABLE I. Presenting a list of the growth parameters of samples, namely, CT-102, CT-103, and CT-105. The thickness of CdTe nucleation layers was approximately $90 \mathrm{~nm}$ for all samples.

\begin{tabular}{|c|c|c|c|c|}
\hline & & Deoxidation & Nucleation & Growth \\
\hline Sample No & II/VI Ratio & $\begin{array}{l}\mathrm{T}\left({ }^{\circ} \mathrm{C}\right) / \text { Time } \\
\quad(\text { min. })\end{array}$ & $\begin{array}{c}\mathrm{T}\left({ }^{\circ} \mathrm{C}\right) / \text { Time } \\
\text { (min.) }\end{array}$ & $\begin{array}{c}\mathrm{T}\left({ }^{\circ} \mathrm{C}\right) / \text { Time } \\
(\text { min. })\end{array}$ \\
\hline CT-102 & 1.0 & $604 / 4$ & $215 / 5$ & $297 / 150$ \\
\hline CT-103 & 1.0 & $613 / 10$ & $226 / 5$ & $299 / 150$ \\
\hline CT-105 & 3.5 & $605 / 4$ & $222 / 5$ & $295 / 130$ \\
\hline
\end{tabular}


beakers at room temperature under fume hood. The chemical reaction that takes place during etch pit formation consists of oxidation and dissolution of the oxide steps. Here, the volume ratios of the constituents of the etching solution determine the rate of etching. In this experiment, the etch solution has been implemented for about $30 \mathrm{~s}$. For the etching, a diced piece of the sample was dipped horizontally into the Everson solution with gentle stirring at room temperature. After that, the samples were rinsed with DI water and then dried under high purity nitrogen gas $\left(\mathrm{N}_{2}\right)$.

\section{High resolution X-ray reciprocal space mapping (RSM)}

The RSM measurements were carried out by means of a four-circle Philips MRD X-ray diffractometer operated at $45 \mathrm{kV}$ and $40 \mathrm{~mA}$. The combination of a four-fold (220) Ge monochromator on the incident beam side and a threefold (220) Ge analyzer on the diffracted beam side was used to set up a high-resolution triple axis configuration. A crossed-slit collimator was used to deliver the incident $\mathrm{Cu} \mathrm{K}_{\alpha 1} \mathrm{X}$-ray beam $(\lambda=1.54 \AA)$ with a spot size of $1.0 \mathrm{~mm} \times 1.0 \mathrm{~mm}$. Scattered $\mathrm{X}$-rays were counted with a Xe-filled gas proportional counter. The symmetric (422) and the asymmetric (511) Bragg reflections of CdTe epilayers were recorded in the $(0 \overline{1} 1)$ diffraction plane. In order to perform these measurements, the radial scans $(\omega-2 \theta)$ were combined with omega $(\omega)$ scans in a coplanar diffraction geometry. Five measurement points per sample were selected to obtain polar contour graphs. The peak widths of the (422) and (511) RLPs were repeatedly measured at all measurement points. The total RSM data collection time per sample was approximately $24 \mathrm{~h}$ in this study.

The physical basis of how the RSM technique determines the density of screw TDs is based on the Bragg peak widths of CdTe epilayers. ${ }^{26}$ In particular, a Burgers vector of $\vec{b}=\mathrm{a} / 6\langle 211\rangle$ can distort all the (h11) Bragg planes in the case of CdTe/(211)B GaAs, so that the measured RSMs in the vicinity of the (511) reflections can be used to estimate the tilt and the lateral coherence length. The extraction of both of these parameters from the asymmetric RLPs is explained in detail elsewhere. ${ }^{16}$ After the estimation, the screw TD densities can be calculated according to $\mathrm{N}_{s}=\beta^{2} / 4.36|\vec{b}|^{2}$. Here, $\beta$ and $|\vec{b}|$ are the tilt and the length of the Burgers vector $(|\vec{b}|=0.265 \mathrm{~nm})$, respectively. ${ }^{18,19}$ The reciprocal space maps for our samples were performed in which $\omega / 2 \theta$ scans were recorded with a step size of $0.006^{\circ}$. The measurement configuration results in highresolution reciprocal space maps with a resolution of approximately $10^{-3} \AA^{-1}$, which allowed us to determine the density of screw-type TDs with a two digit decimal accuracy. Furthermore, the shapes of the (422) RLPs at different measurement points are discussed at the end of this study.

\section{Imaging of CdTe epilayer surfaces}

AFM, carried out using a Solver-Pro 7 system operating in the semi-contact mode, was used to image the sample surface topography and to measure the surface roughnesses of CdTe thin films. It can be noted that all the AFM images were acquired from the samples that were not exposed to the etching solution. These images were taken at $35 \mu \mathrm{m} \times 35 \mu \mathrm{m}$ at room temperature, and image processing was achieved with the help of a software (NT-MDT Nova) in order to obtain optimal viewing of surfaces. For each specimen, the
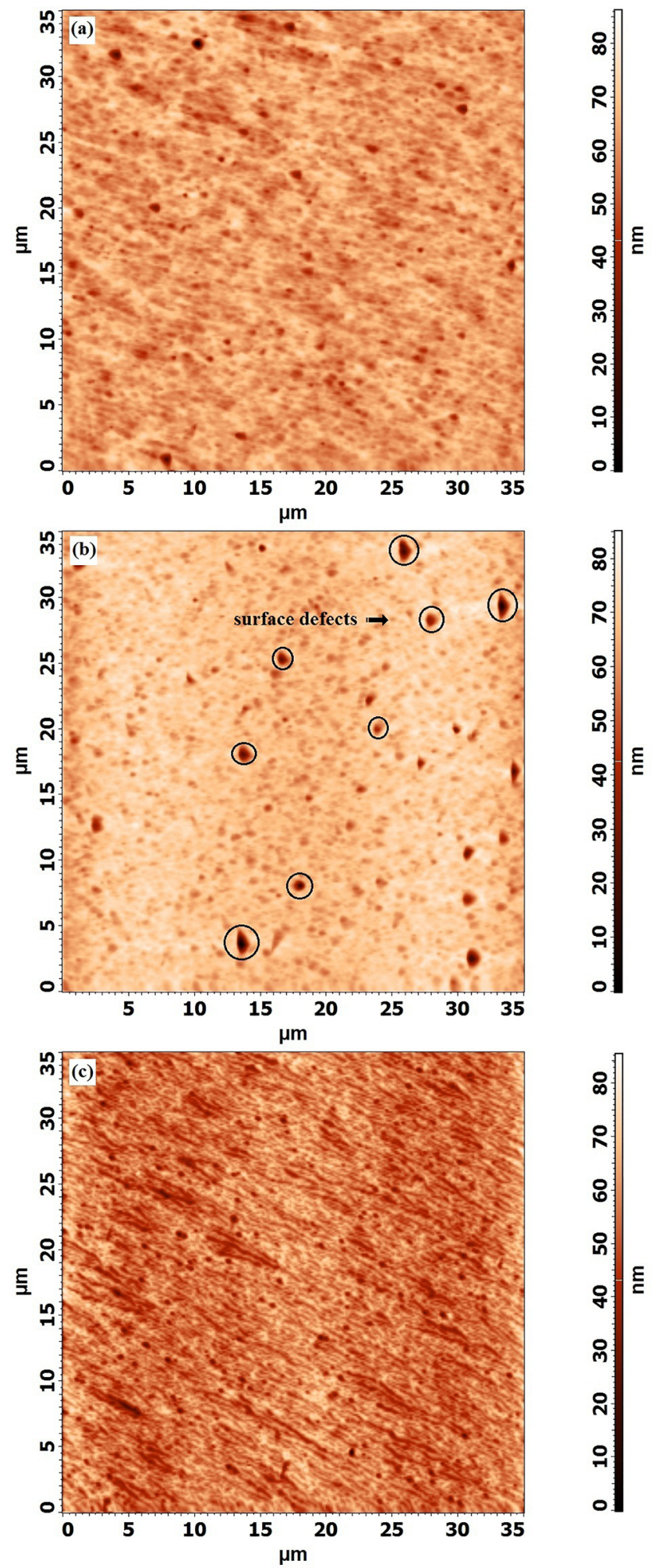

FIG. 1. The AFM 2D topography images of (a) CT-102, (b) CT-103, and (c) CT-105. These $35 \times 35 \mu^{2}$ AFM scans were used to determine the RMS roughnesses of CdTe films before application of the Everson etching method. The RMS roughness values were estimated to be $5.3 \mathrm{~nm}, 5.5 \mathrm{~nm}$, and $4.4 \mathrm{~nm}$, respectively. Some of the surface defects are marked by empty circles. 
TABLE II. Summary of selected specimens. Here, the overall TDs (EPD) determined by the Everson etching method, the screw TD densities calculated with the help of the RSM technique, the ratio stands for the relationship between the total amount of EPD and $\mathrm{N}_{s}$, and the thicknesses measured by FTIR are given.

\begin{tabular}{|c|c|c|c|c|c|}
\hline Sample No & Thickness $(\mu \mathrm{m})$ & Roughness (nm) & $\operatorname{EPD}\left(\times 10^{6} \mathrm{~cm}^{-2}\right)$ & $\mathrm{N}_{s}\left(\times 10^{6} \mathrm{~cm}^{-2}\right)$ & Ratio (\%) \\
\hline CT-102 & 2.7 & 5.3 & 3.51 & 3.18 & 90.6 \\
\hline CT-103 & 2.7 & 5.5 & 14.5 & 13.7 & 94.4 \\
\hline CT-105 & 3.0 & 4.4 & 13.8 & 8.39 & 60.8 \\
\hline
\end{tabular}

root mean square (RMS) values were estimated as the average of three measurements on the surface of the samples.

SEM was performed to observe and to calculate the areal density of etch pits (EPD). The SEM images of the etched CdTe surface were obtained with an accelerating voltage of $10 \mathrm{keV}$, at a magnification of $25000 \times$. Prediction of EPD was based on counting the number of pits by a software program (ImageJ) from the collected SEM images. It is assumed that the number of etch pits per unit area on the etched surface corresponds to the overall TD density throughout this study.

\section{RESULTS AND DISCUSSION}

Surface morphologies of CdTe epilayers closely characterized by AFM are presented in Figs. 1(a)-1(c). It is not easy to determine the types of threading dislocations from these AFM topography images with scanning sizes of $35 \mu \mathrm{m} \times 35 \mu \mathrm{m}$. However, surface defects, marked by empty circles, are easily noticeable, especially in Fig. 1(b). It is known that the presence of the surface defects is a result of the lack of optimal CdTe growth conditions. These surface defects are mainly formed from discrete voids and tellurium precipitates. $^{28}$ The RMS surface roughness values of the specimens of CT102, CT103, and CT105 were estimated to be $5.3 \mathrm{~nm}, 5.5 \mathrm{~nm}$, and $4.4 \mathrm{~nm}$ (Table II), respectively. These values, indicative of good surface quality, are in quite good agreement with those reported in the literature. ${ }^{28}$

In Fig. 2, triangle shapes of the etch pits, usually observed in CdTe epilayers, ${ }^{9}$ are clearly visible in the SEM images. The size of pits can be used to determine the type of dislocations, i.e., the largest and the smallest pits can be associated with the screw-type and the edge-type dislocations, respectively. ${ }^{29}$ However, due to different sized surface pits observed on the surface of the CdTe films (see Fig. 2), we assume that these EPD values give us the total TD density for each sample. The measured values of triangle etch pits for CT102, CT103, and CT105 epilayers were found to be $3.51 \times 10^{6} \mathrm{~cm}^{-2}, 1.45 \times 10^{7} \mathrm{~cm}^{-2}$, and $1.38 \times 10^{7} \mathrm{~cm}^{-2}$, respectively. The total TD densities are in good agreement with literature values. ${ }^{9}$

The density of TDs with screw-component character in (211)-oriented CdTe films was calculated by means of the FWHM(s) data of asymmetric RLPs. The FWHM(s) of the (511) Bragg reflections recorded in the (011) diffraction plane were utilized to predict the tilt and the lateral coherence length. A classic formula $\left(\mathrm{N}_{s}=\beta^{2} / 4.36|\vec{b}|^{2}\right)$ was then employed to determine the screw dislocation density $\left(\mathrm{N}_{s}\right)$ using the tilt. Additionally, high resolution X-ray RSM was carried out at a predetermined number of multiple measurement points of a series of CdTe films grown by MBE under slightly different growth conditions to evaluate the distribution of the screw dislocation density. Figure 3(a) shows variation of density of screw TDs for the specimen of CT102, where the color of the bar represents the number of
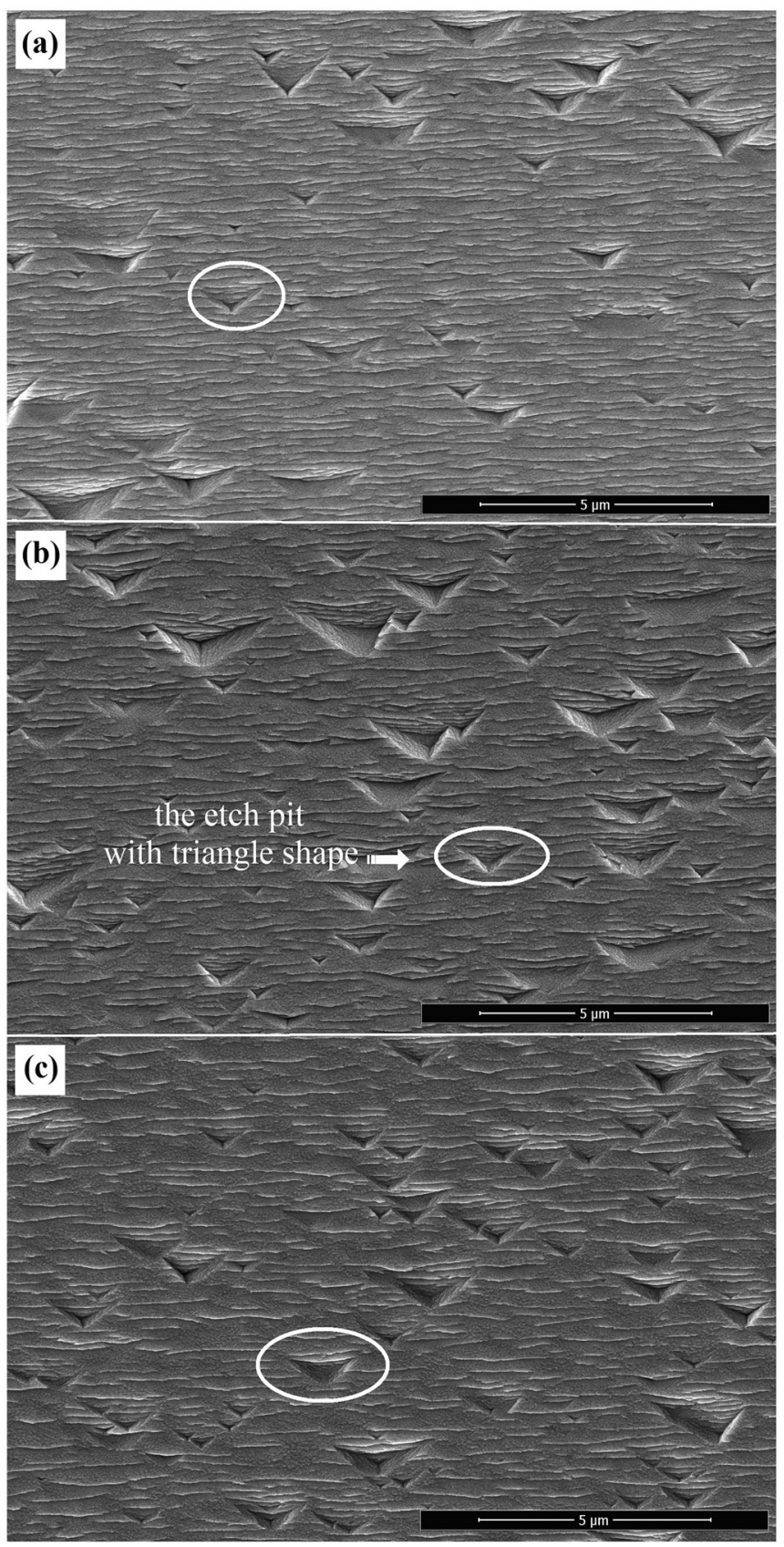

FIG. 2. The SEM images clearly showing etch pits of (a) CT-102, (b) CT103, and (c) CT-105. Magnification is $25000 \times$, and the scale bar corresponds to $5 \mu \mathrm{m}$ for all images. The areal density of etch pits was determined by a software program from these images. 

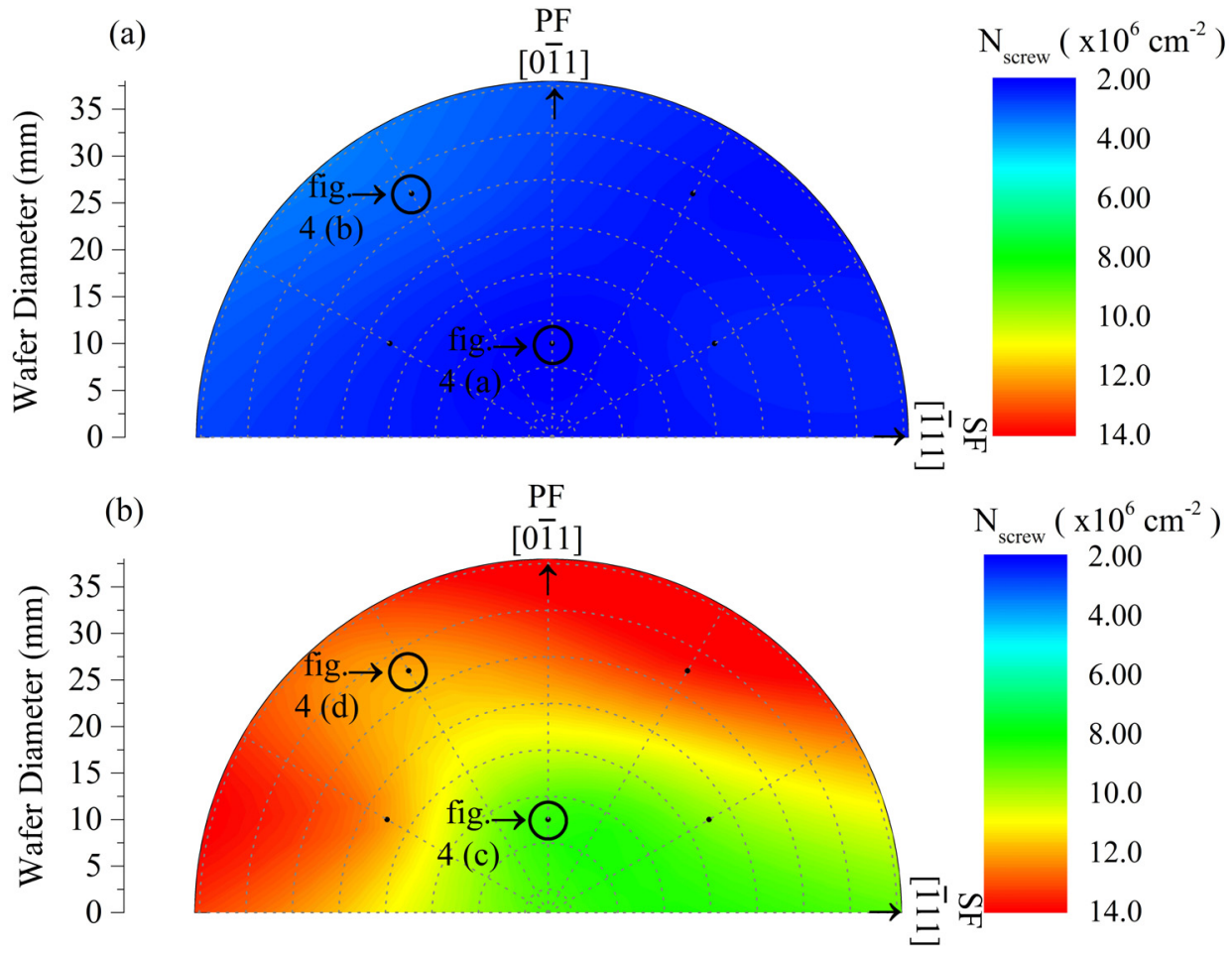

(c)

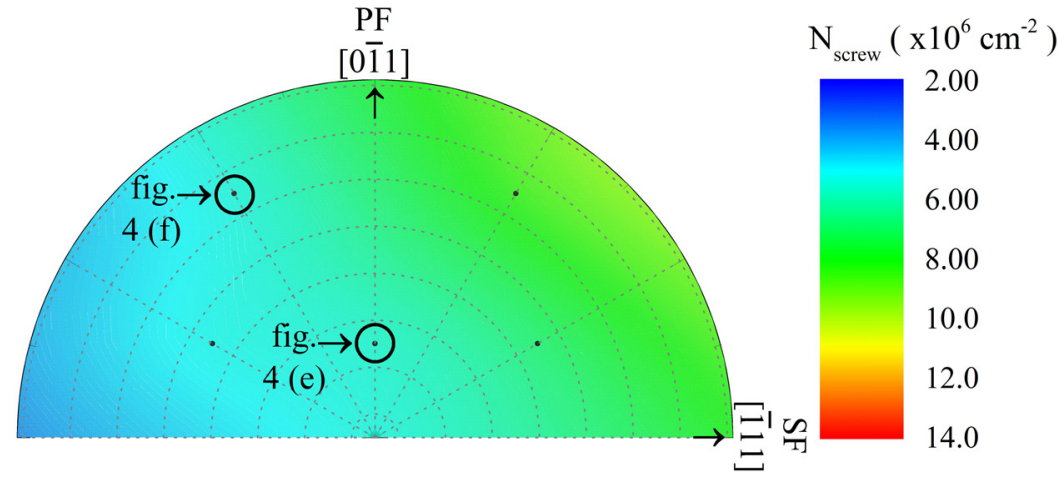

FIG. 3. Polar contour graphs showing the $\mathrm{N}_{s}$, as well as their size and the shapes of CdTe samples (a) CT-102, (b) CT-103, and (c) CT-105. Colours and colour bars on the right demonstrate the dislocation distribution and the densities of the screw TDs, respectively. Primary flats (PF) in the [011] direction and secondary flats $(\mathrm{SF})$ in the [111] direction are represented by arrows in the these graphs. Five selected data points per sample are clearly indicated by black dots in the (011) diffraction plane. Data points, marked by empty circles, show the locations of the (422) RLPs examined in Fig. 4.
TDs with screw component per unit area of the CdTe film, and the arrows show primary flat (PF) and secondary flat $(\mathrm{SF})$ in the [0 $\overline{1} 1]$ and [ $\overline{1} 11]$ directions, respectively. In addition, measurement points are clearly indicated by black dots in the $(0 \overline{1} 1)$ diffraction plane. It was found that the estimated density of TDs with screw-component character for the specimen of CT102 varies in the range from 2.0 $\times 10^{6} \mathrm{~cm}^{-2}$ to $3.18 \times 10^{6} \mathrm{~cm}^{-2}$, and the average lateral coherence length was estimated to be $167 \mathrm{~nm}$. Here, the average lateral coherence length is related to the average extension of the crystal lattice that is free from the defects. Variation of the screw dislocation density across the whole surface and the size and the shape of the CT102 thin film can be easily followed from Fig. 3(a). The polar contour graph highlights that the lower side of the sample has a slightly lower density of screw TD density than the upper side. The overall TD density of the same sample, determined by the SEM images, was counted to be $3.51 \times 10^{6} \mathrm{~cm}^{-2}$. The density of TDs with screwcomponent character seems to account for slightly greater than $90 \%$ of the total TD density, see Table II. The results indicate that the total TD density was dominated by the screw dislocation density for this particular sample.
The distribution of the density of TDs with screwcomponent character corresponding to the specimen of CT103 is shown in Fig. 3(b). Note that the spatial variations in this polar contour graph are nonuniform. Based on the distribution in Fig. 3(b), we can conclude that the upper side contains the much higher TD density than the lower side of the sample. This can be due to the nonuniform heating of the GaAs wafer or the effect of the residual stresses in the epilayer. The density of TDs with screw-component character ranges from $8.44 \times 10^{6} \mathrm{~cm}^{-2}$ to $13.7 \times 10^{6} \mathrm{~cm}^{-2}$, and hence, we decide to use the value of $13.7 \times 10^{6} \mathrm{~cm}^{-2}$ in Table II since this value is dominant for the CT103 epilayer. The average lateral coherence length was calculated to be $83 \mathrm{~nm}$. The smaller lateral coherence length value corresponds to higher dislocation density in the CdTe epilayer grown on the (211)-oriented GaAs substrate. The total TD density is found to be $14.5 \times 10^{6} \mathrm{~cm}^{-2}$ with the help of SEM images. For the epilayer of CT103, the overall TD density contains a large percentage, i.e., $94.4 \%$, of the density of TDs with screwcomponent character. Therefore, it is reasonable to conclude that the screw dislocation density is the dominant dislocation type in the CT103 epilayer. A previous study, ${ }^{7}$ carried out by TEM, stated that the edge component TDs accounted for 
only $2.4 \%$ of the total TD density for CdTe thin films grown by MBE, and this shows a good agreement with our results. The number of both TDs with the screw component and the overall TD per unit area of the CT103 epilayer is roughly four times larger as compared to those estimated for the specimen of CT102. This result probably points out that the CT103 epilayer grown at high nucleation temperature, i.e., $226^{\circ} \mathrm{C}$, tends to have a higher overall TD density than the specimen of CT102 and also a higher density of screw TDs than the CT102 epilayer deposited at lower nucleation temperatures, i.e., $215^{\circ} \mathrm{C}$. As we described above, the aim of growing CdTe nucleation layers $(\sim 90 \mathrm{~nm})$ on GaAs surfaces at relatively low temperatures is to trigger the occurrence of the (211) epitaxial relationship prior to epilayer growth. When the nucleation temperatures are sufficiently low, the lattice parameters of these nucleation layers slightly shrink or expand depending on these temperatures. Therefore, the CT102 epilayer, deposited at lower nucleation temperature, has a lattice constant that is a bit smaller than that of the CT103 epilayer. As a result, the specimen of CT102 has a smaller defect-density value due to its relatively low lattice mismatch with the GaAs substrate as compared to the CT103 epilayer.

Figure 3(c) clearly displays a significant difference in densities of screw-type TDs from one side of the CT105 epilayer to the other. It was lower, i.e., $5.23 \times 10^{6} \mathrm{~cm}^{-2}$, at the left side, while the density of screw-type TDs was relatively high, i.e., $8.39 \times 10^{6} \mathrm{~cm}^{-2}$, at the right side. In addition, we calculated the average lateral coherence length as $107 \mathrm{~nm}$ for the CT105 epilayer. The total TD density was predicted to be $13.8 \times 10^{6} \mathrm{~cm}^{-2}$, which means that TD densities were dominated by the density of TDs with screw-component character, accounting for slightly greater than $60 \%$ of the total TD density. Analysis of the growth parameters, listed in Table I, indicates that the high II/VI flux ratio of 3.5 causes a decrease in the percentage of the density of screw TDs. It is clear that the II/VI $(\mathrm{Cd} / \mathrm{Te})$ flux ratio can dramatically affect the ratio between the densities of TDs with screwcomponent character and the total TD density. As the flux ratio increases, the density of screw TDs shows a significant decrease, while the total TD density nearly remains identical. We believe that a low flux of the Te element may result in high density edge-type TDs, but further extensive research is necessary to support this finding.

To deeply understand the spatial distribution of screw TD densities, shown in Figs. 3(a)-3(c), the measured RSMs in the vicinity of the (422) reflections are demonstrated. In Figs. 4(a)-4(f), the RSMs around the (422) CdTe Bragg peaks were measured at the upper and lower sides of the thin films. Here, Figs. 4(a), 4(c), and 4(e) display the (422) symmetrical diffraction peaks at the lower sides, while Figs. 4(b), 4(d), and 4(f) were recorded at the upper sides of the specimens of CT102, CT103, and CT105, respectively. The peak widths of the (422) reflections from the [111], shown in Figs. 4(b), 4(d), and 4(f), tend to be more elongated than those measured at the lower sides of the samples. This elongation for all the films implies that the mosaicity is much more dominant at the upper sides. The spatial distribution of the screw TD densities, presented in the polar contour graphs, shows good agreement with this result.

In brief, we apply a method which allows us to perform for fast and relatively reliable structural analysis to determine the density of screw TDs in CdTe thin films grown on (211)oriented substrates. Here, the screw TDs are assumed to be randomly distributed in selected specimens, and a classical formula $\left(\mathrm{N}_{s}=\beta^{2} / 4.36|\vec{b}|^{2}\right)$ is employed for quantification of
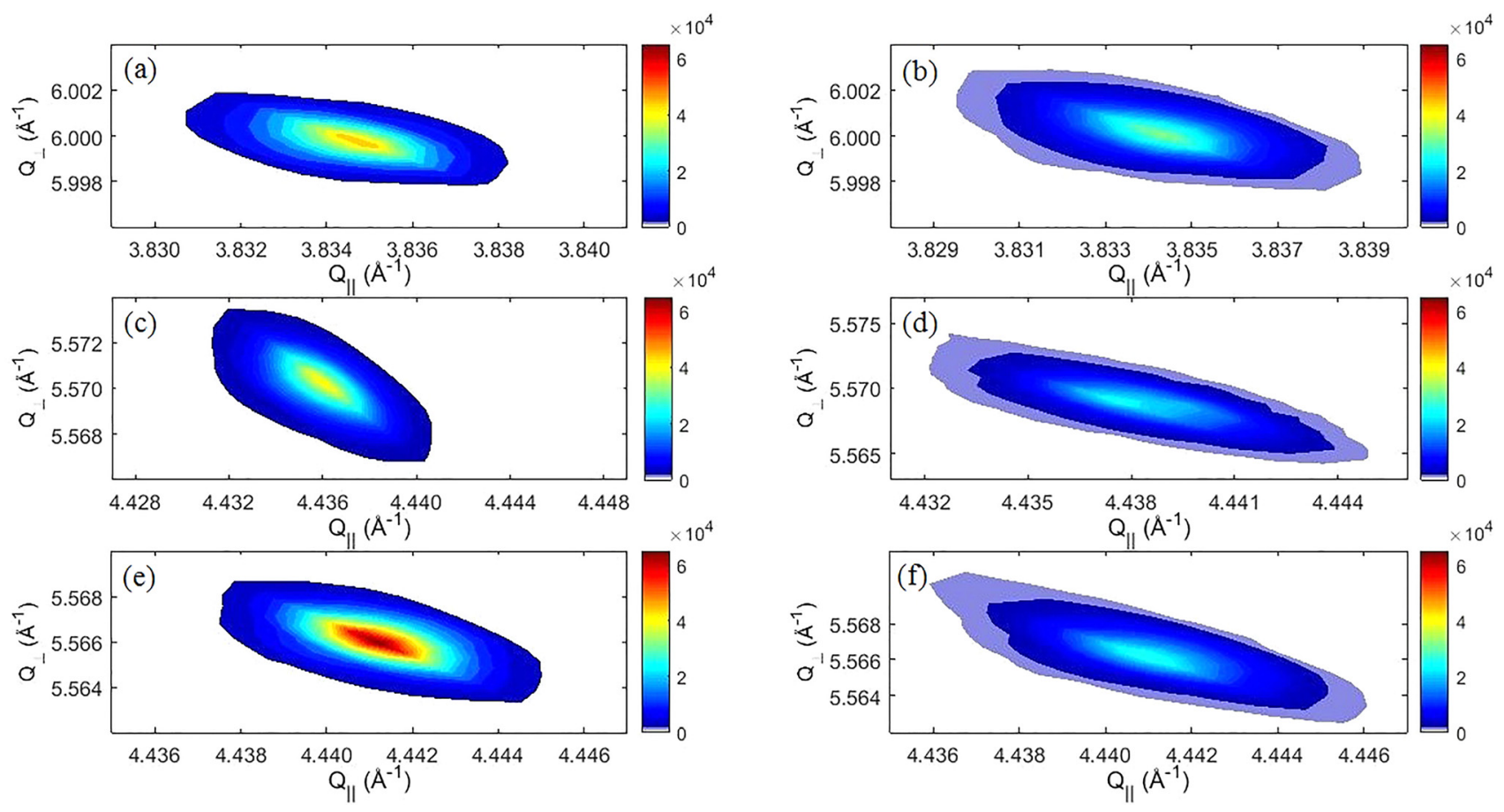

FIG. 4. The shapes of the (422) RLPs from [1111] for (a) and (b) CT-102, (c) and (d) CT-103, and (e) and (f) CT-105. Here, (a), (c), and (e) were recorded at the lower sides of CT-102, CT-103, and CT-105, while (b), (d), and (f) were measured at the upper sides of CT-102, CT-103, and CT-105, respectively. 
the screw TD densities. As suggested by previous research, ${ }^{14}$ XRD probably tends to underestimate the screw TD densities due to experimental factors such as the instrumental broadening of Bragg reflections, a classical formula utilized here, and configuration of epilayer dislocations. The approach presented in this study can be fully specialized for this particular material system by combining XRD and TEM to understand the impact of these factors for a more reliable absolute quantification of the defect density. This method can be refined in this way in order to verify the accuracy of the method, and it is possible to take advantage of the non-destructive nature of XRD.

\section{CONCLUSIONS}

We have studied the TDs for a series of CdTe films grown by MBE under slightly different growth conditions by both RSM and decorative etching. While the defect-induced broadening of asymmetric RSM around the (511) RLPs is used to estimate the screw TDs, we have extracted the total TD density from the Everson-etching method. We found that the dominant dislocation type is the screw dislocation in CdTe films grown on the highly lattice-mismatched GaAs substrate. The screw TDs are responsible for $>90 \%$ of the total TD density of the films. It is also clarified that the epilayers grown at higher nucleation temperatures $\left(\geq 222^{\circ} \mathrm{C}\right)$ tend to be more defective. At the lower nucleation temperature $\left(\leq 215^{\circ} \mathrm{C}\right)$, both the screw TDs and the total TD density are approximately four times smaller than the values measured for the epilayers grown at higher nucleation temperatures $\left(\geq 222^{\circ} \mathrm{C}\right)$. In the case of a high II/VI flux ratio, i.e., 3.5 , the contribution of screw TDs to the total TD density shows a significant decrease in roughly $30 \%$.

We further examined the RSMs around the (422) CdTe Bragg peaks for all the specimens. The widths of the peaks of the measurement points close to the upper sides become broader for all the films, probably indicating that the mosaicity is higher at the upper sides with respect to the lower sides. The reason could be due to temperature differences caused by the bowing of CdTe epitaxial layers.

\section{ACKNOWLEDGMENTS}

This work was supported by the Turkish Ministry of National Defence, the Undersecretariat for Defence Industries (SSM), under project name of GEDIZ and was performed at Izmir Institute of Technology. This paper is dedicated to the memory of Associate Professor Yusuf Selamet at IZTECH, who sadly passed away on Friday, August 5th, 2016.
${ }^{1}$ V. Varavin, V. Vasiliev, S. Dvoretsky, N. Mikhailov, V. Ovsyuk, Y. Sidorov, A. Suslyakov, M. Yakushev, and A. Aseev, Opto-Electron. Rev. 2, 99 (2003), available at https://chemport.cas.org/cgi-bin/ sdcgi? APP=ftslink\&action=reflink\&origin=aip\&version $=1.0 \&$ coi $=1 \% 3$ ACAS\%3A528\%3ADC\%252BD3sXmtFSqu7s\%253D\&md5=eb7faaccacd7fd $23411063502472 \mathrm{fc} 90$.

${ }^{2}$ L. He, L. Chen, Y. Wu, X. Fu, Y. Wang, J. Wu, M. Yu, J. Yang, R. Ding, X. Hu et al., J. Cryst. Growth 301, 268 (2007).

${ }^{3}$ O. Ari, M. Polat, M. Karakaya, and Y. Selamet, Phys. Status Solidi C 12, 1211 (2015).

${ }^{4}$ P. J. Sellin, Nucl. Instrum. Methods Phys. Res., Sect. A 513, 332 (2003).

${ }^{5}$ M. Carmody, A. Yulius, D. Edwall, D. Lee, E. Piquette, R. Jacobs, D. Benson, A. Stoltz, J. Markunas, A. Almeida et al., J. Electron. Mater. 41, 2719 (2012).

${ }^{6}$ R. Jacobs, J. Markunas, J. Pellegrino, L. Almeida, M. Groenert, M. JaimeVasquez, N. Mahadik, C. Andrews, and S. Qadri, J. Cryst. Growth 310, 2960 (2008).

${ }^{7}$ J. J. Kim, R. Jacobs, L. Almeida, M. Jaime-Vasquez, C. Nozaki, and D. J. Smith, J. Electron. Mater. 42, 3142 (2013).

${ }^{8}$ I. Sugiyama, A. Hobbs, T. Saito, O. Ueda, K. Shinohara, and H. Takigawa, J. Cryst. Growth 117, 161 (1992).

${ }^{9}$ E. Bilgilisoy, S. Özden, E. Bakali, M. Karakaya, and Y. Selamet, J. Electron. Mater. 44, 3124 (2015).

${ }^{10}$ X. Zhou, D. K. Ward, B. M. Wong, F. P. Doty, and J. A. Zimmerman, J. Phys. Chem. C 116, 17563 (2012).

${ }^{11}$ P. F. Fewster, Crit. Rev. Solid State Mater. Sci. 22, 69 (1997).

${ }^{12}$ M. Moram and M. Vickers, Rep. Prog. Phys. 72, 036502 (2009).

${ }^{13}$ V. Srikant, J. Speck, and D. Clarke, J. Appl. Phys. 82, 4286 (1997).

${ }^{14}$ S. Lee, A. West, A. Allerman, K. Waldrip, D. Follstaedt, P. Provencio, D. Koleske, and C. Abernathy, Appl. Phys. Lett. 86, 241904 (2005).

${ }^{15}$ R. Chierchia, T. Böttcher, H. Heinke, S. Einfeldt, S. Figge, and D. Hommel, J. Appl. Phys. 93, 8918 (2003).

${ }^{16} \mathrm{P}$. F. Fewster, X-Ray Scattering from Semiconductors (World Scientific, 2003).

${ }^{17}$ M. Birkholz, Thin Film Analysis by X-Ray Scattering (John Wiley and Sons, 2006).

${ }^{18}$ P. Gay, P. Hirsch, and A. Kelly, Acta Metall. 1, 315 (1953).

${ }^{19}$ C. Dunn and E. Kogh, Acta Metall. 5, 548 (1957).

${ }^{20}$ C. Gallinat, G. Koblmüller, F. Wu, and J. Speck, J. Appl. Phys. 107, 053517 (2010).

${ }^{21}$ Ü. H. Heinke, M. Möller, D. Hommel, and G. Landwehr, J. Cryst. Growth 135, 41 (1994).

${ }^{22}$ Y. Ryu, T. Kang, and T. Kim, J. Mater. Sci. 40, 4699 (2005).

${ }^{23}$ R. Jacobs, L. Almeida, J. Markunas, J. Pellegrino, M. Groenert, M. JaimeVasquez, N. Mahadik, C. Andrews, S. Qadri, T. Lee et al., J. Electron. Mater. 37, 1480 (2008).

${ }^{24}$ T. Sasaki, M. Tomono, and N. Oda, J. Vac. Sci. Technol. B 10, 1399 (1992).

${ }^{25}$ C. Lennon, L. Almeida, R. Jacobs, J. Markunas, P. Smith, J. Arias, A. Brown, and J. Pellegrino, J. Electron. Mater. 41, 2965 (2012).

${ }^{26}$ M. Polat, O. Ari, O. Öztürk, and Y. Selamet, Mater. Res. Express 4, 035904 (2017).

${ }^{27}$ M. Jaime-Vasquez, M. Martinka, R. Jacobs, and M. Groenert, J. Electron. Mater. 35, 1455 (2006).

${ }^{28}$ R. Jacobs, C. Nozaki, L. Almeida, M. Jaime-Vasquez, C. Lennon, J. Markunas, D. Benson, P. Smith, W. Zhao, D. Smith et al., J. Electron. Mater. 41, 2707 (2012).

${ }^{29}$ J. Weyher, L. Macht, G. Kamler, J. Borysiuk, and I. Grzegory, Phys. Status Solidi C 0, 821 (2003). 